\title{
Proximity inequalities for complete ideals in two-dimensional regular local rings
}

\author{
JOSEPH LIPMAN
}

0. Introduction

1. Preliminaries

2. Proximity inequalities

3. Unique factorization

4. Simple complete ideals

5. Valuations and proximity

Introduction. Among the various algebraic approaches to the classification of singularities of irreducible plane curves (via characteristic pairs, multiplicity sequence, value semigroup, etc.) a particularly attractive one, based on the idea of proximity, was developed by Enriques [4, book 4]. His analysis can be adapted to arbitrary valuations birationally dominating two-dimensional regular local rings, and also to complete - i.e., integrally closed - ideals in such rings.

While some motivational material on valuations appears in $\S 5$, this paper deals mainly with complete ideals. The principal result, Theorem (2.1), provides a necessary and sufficient condition, the proximity inequalities, for the existence of a complete ideal having a given "point basis." This is the ideal-theoretic version of an old result on the existence of plane curves with given effective multiplicities at infinitely near points, cf. [4, p. 392, p. 427], [13, p. 196, Thm. 14], [8, p. 49]. Unique factorization for complete ideals and some basic properties of simple complete ideals fall out as corollaries, more or less. Theorem (4.11), on the predecessor of a simple complete ideal, is inspired by [5, $\S 3]$, but says more.

Basically then, our purpose is to publicize the efficacy of the notion of proximity, and in particular to redo some of Zariski's theory of complete ideals ${ }^{1}$ so as to expose further its roots in the classical treatment of the local behavior of linear systems of curves on smooth surfaces .

1991 Mathematics Subject Classification. 13H05, 14H20.

Partially supported by the National Security Agency.

${ }^{1}$ cf. [15, Appendix 5], [7, chaps. II, V], [9], [6]. 
1. Preliminaries. (1.1) Fix a field $K$, and denote by $\alpha, \beta, \gamma, \ldots$ twodimensional regular local rings - which we call "points" — having fraction field $K$.

To connect with classical language, we say that a point $\beta$ is "infinitely near" to a point $\alpha$ if $\beta \supset \alpha$. Then the maximal ideal $\mathfrak{m}_{\beta}$ of $\beta$ intersects $\alpha$ in $\mathfrak{m}_{\alpha}$. Moreover, a factorization theorem of Zariski and Abhyankar [1, p. 343, Thm. 3] gives the existence of a finite sequence - clearly unique-

$$
\alpha=\alpha_{0} \subset \alpha_{1} \subset \cdots \subset \alpha_{n}=\beta
$$

such that for $0 \leq i<n, \alpha_{i+1}$ is a quadratic transform of $\alpha_{i}$, i.e., a localization at a maximal ideal of a ring $\alpha_{i}\left[x^{-1} \mathfrak{m}_{\alpha_{i}}\right]$ with $x \in \mathfrak{m}_{\alpha_{i}}, x \notin \mathfrak{m}_{\alpha_{i}}^{2}$. So the residue field extension $\alpha / \mathfrak{m}_{\alpha} \subset \beta / \mathfrak{m}_{\beta}$ is finite; we denote its degree by $[\beta: \alpha]$.

(1.2) To each point $\beta$ associate the unique valuation $\operatorname{ord}_{\beta}$ of $K$ such that

$$
\operatorname{ord}_{\beta}(x)=\max \left\{n \mid x \in \mathfrak{m}_{\beta}^{n}\right\} \quad(0 \neq x \in \beta) .
$$

This association is a one-one correspondence between points infinitely near to a given point $\alpha$ and valuations $v$ of $K$ dominating and residually transcendental over $\alpha$, the point associated to such a $v$ being the largest one containing $\alpha$ and dominated by $v[\mathbf{1}$, p. 336, Prop. 3].

(1.3) We say that $\beta \supsetneqq \alpha$ is proximate to $\alpha$, and write $\beta \succ \alpha$, if the valuation ring of $\operatorname{ord}_{\alpha}$ contains $\beta$-and hence is of the form $\beta_{\mathfrak{p}}$ where $\mathfrak{p}$ is a height one prime ideal in $\beta$ containing $\mathfrak{m}_{\alpha}$.

An easy induction on the length $n$ of the sequence (1.1.1) shows that if $\beta \supsetneqq \alpha$ then $\mathfrak{m}_{\alpha} \beta=t^{a} u^{b} \beta$, where $t \beta=\mathfrak{m}_{\alpha_{n-1}} \beta,(t, u) \beta=\mathfrak{m}_{\beta}, a>0$ and $b \geq 0$. Consequently $\beta$ is proximate to $\alpha_{n-1}$ and to at most one other point in (1.1.1).

Thinking geometrically, consider a map $f: X \rightarrow Y$ of smooth surfaces, let $\alpha$ be the local ring of a point $y \in Y$, and let $\beta$ be the local ring of a point $x \in f^{-1}(y)$; then the points to which $\beta$ is proximate correspond to the components of $f^{-1}(y)$ through $x$-at most two, since $f^{-1}(y)$ is a normal-crossing divisor.

(1.4) The integral closure $\bar{I}$ of an ideal $I$ in $\alpha$ satisfies

$$
\bar{I}=\left\{x \in \alpha \mid \operatorname{ord}_{\beta}(x) \geq \operatorname{ord}_{\beta}(I) \text { for all } \beta \supset \alpha\right\} .
$$

It is actually enough here to consider only those $\beta$ such that $\operatorname{ord}_{\beta} \in \mathbf{R}(I)$, the set of Rees valuations of $I$, i.e., those valuations which correspond to the components of the closed fiber on the normalized blowup of $I$, cf. e.g., [15, p. 354, Lemma].

Conforming with Zariski's terminology, we say that $I$ is complete if $I=\bar{I}$.

The product of any two complete ideals is complete $\left[\mathbf{1 5}, \mathrm{p} .385\right.$, Thm. $\left.2^{\prime}\right] .^{2}$

(1.5) Let $I$ be an $\alpha$-ideal of finite colength, i.e., $\lambda_{\alpha}(\alpha / I)<\infty$ (where $\lambda_{\alpha}$ denotes the length of an $\alpha$-module). The transform of $I$ in a point $\beta \supset \alpha$ is the finite-colength $\beta$-ideal $I^{\beta}:=I(I \beta)^{-1}$. Note that $I \beta=t^{c} u^{d} I^{\beta}$ where $t$ and $u$ are as in (1.3), $c=\operatorname{ord}_{\gamma_{1}}(I)$ with $\gamma_{1}$ the predecessor of $\beta$ in the sequence (1.1.1), and $d=\operatorname{ord}_{\gamma_{2}}(I)$ with $\gamma_{2}$ the other point in (1.1.1) to which $\beta$ is proximate (if there is one; otherwise $d=0$ ).

\footnotetext{
${ }^{2}$ For a generalization to rational singularities, cf. [7, p. 209, Thm. (7.1)].
} 
Transform preserves products: $(I J)^{\beta}=I^{\beta} J^{\beta}$.

The transform operation is transitive: if $\alpha \subset \beta \subset \gamma$ then $\left(I^{\beta}\right)^{\gamma}=I^{\gamma}$.

(1.6) $\beta \supset \alpha$ is a base point of $I$ if $\operatorname{ord}_{\beta}\left(I^{\beta}\right) \neq 0$, i.e., if $I \beta$ is not a principal ideal. A given $\alpha$-ideal $I$ has only finitely many base points, since any such $\beta$ is dominated by a Rees valuation of $I$ (as follows from Zariski's Main Theorem, because $\beta$ does not contain any local ring on the normalized blowup of $I$ ).

The point basis of $I$ is the family of nonnegative integers

$$
\mathbf{B}(I):=\left(\operatorname{ord}_{\beta}\left(I^{\beta}\right)\right)_{\beta \supset \alpha} .
$$

For any two finite-colength $\alpha$-ideals $I, J$, we have:

(i) $\mathbf{B}(I J)=\mathbf{B}(I)+\mathbf{B}(J) \quad$ (since $(I J)^{\beta}=I^{\beta} J^{\beta}$ )

(ii) $\bar{I}=\bar{J} \Longleftrightarrow \mathbf{B}(I)=\mathbf{B}(J) \quad([\mathbf{9}$, p. 209, (1.10)]).

Thus $\mathbf{B}$ maps the multiplicative monoid $\mathcal{M}_{\alpha}$ of finite-colength complete $\alpha$-ideals isomorphically onto a submonoid $\mathbf{B}\left(\mathcal{M}_{\alpha}\right)$ of the free commutative monoid generated by all the points infinitely near to $\alpha$.

The following central result describes $\mathbf{B}\left(\mathcal{M}_{\alpha}\right)$.

\section{Proximity inequalities.}

THEOREM (2.1). Let $\left(r_{\beta}\right)_{\beta \supset \alpha}$ be a family of nonnegative integers, with $r_{\beta}=0$ for all but finitely many $\beta$. Then there exists a finite-colength $\alpha$-ideal $I$ with $\mathbf{B}(I)=\left(r_{\beta}\right)$ iff the following proximity inequality holds for each $\beta \supset \alpha$ :

$$
r_{\beta} \geq \sum_{\gamma \succ \beta}[\gamma: \beta] r_{\gamma}
$$

And if there is such an I then there is one and only one which is complete.

The second assertion follows from (1.6)(ii); the proof of the first takes up the rest of this section. (See also the remark at the very end of the paper.) We'll say that an $\alpha$-ideal $I$ is divisible by an $\alpha$-ideal $J$ if $I=J J^{\prime}$ for some $\alpha$-ideal $J^{\prime}$ (or, equivalently, if $I=J(I: J)$ ). A key point is:

Lemma (2.2). Let I be a finite-colength complete $\alpha$-ideal, and let $\nu \geq 0$ be the integer such that $I$ is divisible by $\mathfrak{m}_{\alpha}^{\nu}$ but not by $\mathfrak{m}_{\alpha}^{\nu+1}$. Then

$$
\nu=\operatorname{ord}_{\alpha}(I)-\sum_{\gamma \succ \alpha}[\gamma: \alpha] \operatorname{ord}_{\gamma}\left(I^{\gamma}\right) .
$$

Assuming (2.2), whose proof will be given below, we can prove (2.1) as follows.

Suppose that $\left(r_{\beta}\right)=\mathbf{B}(I)$. To prove the inequality for $r_{\beta}:=\operatorname{ord}_{\beta}\left(I^{\beta}\right)$, we can simply replace $\alpha$ by $\beta$ and $I$ by $I^{\beta}$ in (2.2) and apply transitivity of transform (1.5).

Suppose conversely that the family $\left(r_{\beta}\right)$ satisfies the proximity inequalities. Let $\beta_{1}, \beta_{2}, \ldots, \beta_{n}(n \geq 0)$ be all those quadratic transforms of $\alpha$ whose corresponding $r$ doesn't vanish. Inducting on the number of $\beta$ such that $r_{\beta} \neq 0$, we may assume that there exists a finite-colength complete $\beta_{j}$-ideal $I_{j}$ with point 
basis $\left(r_{\beta}\right)_{\beta \supset \beta_{j}}$. By $[\mathbf{9}$, p. 217, Lemma (2.3)] there is a finite-colength complete $\alpha$-ideal $I^{\prime}$, not divisible by $\mathfrak{m}_{\alpha}$, whose transform in $\beta_{j}$ is $I_{j}(1 \leq j \leq n)$ and whose transform in every other quadratic transform of $\alpha$ is the unit ideal. By assumption,

$$
0 \leq r_{\alpha}-\sum_{\gamma \succ \alpha}[\gamma: \alpha] r_{\gamma} \stackrel{(2.2)}{=} r_{\alpha}-\operatorname{ord}_{\alpha}\left(I^{\prime}\right)=: a,
$$

so we may set $I:=\mathfrak{m}_{\alpha}^{a} I^{\prime} ;$ and then $\mathbf{B}(I)=\left(r_{\beta}\right)$.

Now here is the proof of (2.2). Set $\mathfrak{m}:=\mathfrak{m}_{\alpha}, k:=\alpha / \mathfrak{m}$. If $I=\mathfrak{m}^{e} J(e \geq 0)$ then $I^{\gamma}=J^{\gamma}$ for all $\gamma \supsetneqq \alpha$, so that the sum $\sum_{\gamma \succ \alpha}[\gamma: \alpha] \operatorname{ord}_{\gamma}\left(I^{\gamma}\right)$ does not change when $I$ is replaced by $J$; and it follows that for (2.2) it suffices to treat the case where $\nu=0$.

Let $r:=\operatorname{ord}_{\alpha}(I)$, so that $I \subset \mathfrak{m}^{r}, I \nsubseteq \mathfrak{m}^{r+1}$. Let $s(I)$ be the degree of the greatest common divisor $c(I)$ (in the graded UFD $\operatorname{gr}(\alpha):=\oplus_{n \geq 0} \mathfrak{m}^{n} / \mathfrak{m}^{n+1}$ ) of all the elements in the initial form vector space

$$
\operatorname{in}(I):=\left(I+\mathfrak{m}^{r+1}\right) / \mathfrak{m}^{r+1} \subset \mathfrak{m}^{r} / \mathfrak{m}^{r+1} \subset \operatorname{gr}(\alpha) .
$$

A basic result of Zariski [15, p. 368, Prop.3], [6, p. 327, Prop. 2.5] is that if I is not divisible by $\mathfrak{m}$ then $s(I)=\operatorname{ord}_{\alpha}(I)$. Thus (2.2) (for $\nu=0$, and hence for all $\nu$ ) follows from the next result, which is an ideal-theoretic analog of $[\mathbf{1 3}$, p. 191, Thm. 11]:

Proposition (2.3). For any finite-colength $\alpha$-ideal I, we have

$$
s(I)=\sum_{\gamma \succ \alpha}[\gamma: \alpha] \operatorname{ord}_{\gamma}\left(I^{\gamma}\right) .
$$

Proof. The proof uses a family $v_{p}$ of valuations of $K$, one for each homogeneous height one prime ideal $p$ in $\operatorname{gr}(\alpha)$. For any nonzero $x \in \alpha$, let

$$
\bar{x}:=x+\mathfrak{m}^{b+1} \in\left(\mathfrak{m}^{b} / \mathfrak{m}^{b+1}\right) \subset \operatorname{gr}(\alpha) \quad\left(b:=\operatorname{ord}_{\alpha}(x)\right)
$$

be the initial form of $x$. Since $\operatorname{gr}(\alpha)$ is isomorphic to a polynomial ring over the residue field $k$, we can factor the principal ideal $(\bar{x})$ uniquely in the form

$$
(\bar{x})=\prod_{p} p^{n_{p}(x)} .
$$

It is then easily checked that the mapping $x \mapsto\left(\operatorname{ord}_{\alpha}(x), n_{p}(x)\right)$ of $\alpha$ into the lexicographically ordered group $\mathbb{Z} \times \mathbb{Z}$ gives rise to a valuation $v_{p}$ of $K$ which dominates $\alpha$ and is composite with $\operatorname{ord}_{\alpha}$ (i.e., its valuation ring is contained in that of $\operatorname{ord}_{\alpha}$ ). There is a unique quadratic transform $\beta_{p}$ of $\alpha$ which is dominated by $v_{p}$; and one verifies that $p \mapsto \beta_{p}$ is a one-one correspondence between the set of homogeneous height one primes $p$ and the set of quadratic transforms of $\alpha$ (both sets corresponding to the set of closed points in the closed fiber of the blowup of $\mathfrak{m}$.) Note that if a point $\gamma$ is contained in the valuation ring $V_{p}$ of $v_{p}$, then $v_{p}$ dominates $\gamma$ : the center of $v_{p}$ in $\gamma$-i.e., the intersection $\mathfrak{q}$ of $\gamma$ with the maximal ideal of $V_{p}$-is $\mathfrak{m}_{\gamma}$ (because $V_{p}$ contains the localization $\gamma_{\mathfrak{q}}$ ). 
The significance of the $v_{p}$ with respect to proximity is given by:

Lemma (2.4). (i) If $\alpha \subset \gamma \subset V_{p}$, then $\gamma$ contains $\beta_{p}$ and is proximate to $\alpha$.

(ii) Conversely, if $\gamma$ contains $\beta_{p}$ and is proximate to $\alpha$, then $\gamma \subset V_{p}$. In fact $V_{p}$ is the unique valuation ring dominating $\gamma$ and composite with $\operatorname{ord}_{\alpha}$, i.e., contained in the valuation ring $R$ of $\operatorname{ord}_{\alpha}$. Moreover, the inclusion $\gamma \hookrightarrow V_{p}$ induces an isomorphism of residue fields, and $v_{p}\left(\mathfrak{m}_{\gamma}\right)=(0,1)$.

Before proving (2.4), let us deduce (2.3). Factoring the gr $(\alpha)$-ideal $(c(I))$ as

$$
(c(I))=\prod_{p} p^{n_{p}(I)}
$$

and observing that $p$ is generated by a homogeneous element of degree $\left[\beta_{p}: \alpha\right]$, we reduce to showing that

$$
n_{p}(I)=\sum_{\substack{\gamma \supset \beta_{p} \\ \gamma \succ \alpha}}\left[\gamma: \beta_{p}\right] \operatorname{ord}_{\gamma}\left(I^{\gamma}\right) .
$$

In view of (2.4), which implies in particular that $\gamma$ and $\beta_{p}$ have the same residue field, i.e., $\left[\gamma: \beta_{p}\right]=1$, the right hand sum becomes $\sum_{V_{p} \supset \gamma \supsetneq \alpha} \operatorname{ord}_{\gamma}\left(I^{\gamma}\right)$. But

$$
\left.\operatorname{ord}_{\alpha}(I), n_{p}(I)\right)=v_{p}(I)=\sum_{V_{p} \supset \gamma \supset \alpha} \operatorname{ord}_{\gamma}\left(I^{\gamma}\right) v_{p}\left(\mathfrak{m}_{\gamma}\right)
$$

(For the second equality use, repeatedly, the fact that with $\delta$ the unique quadratic transform of $\gamma$ dominated by $v_{p}$, we have $I^{\gamma} \delta=\mathfrak{m}_{\gamma}^{\operatorname{ord}_{\gamma}\left(I^{\gamma}\right)} I^{\delta}$, so that

$$
v_{p}\left(I^{\gamma}\right)=v_{p}\left(I^{\delta}\right)+\operatorname{ord}_{\gamma}\left(I^{\gamma}\right) v_{p}\left(\mathfrak{m}_{\gamma}\right)
$$

cf. $\left[\mathbf{9}\right.$, p. 209, Lemma (1.11)].) Since $v_{p}\left(\mathfrak{m}_{\alpha}\right)=(1,0)$ and since (by (2.4)) $v_{p}\left(\mathfrak{m}_{\gamma}\right)=(0,1)$ for all $\gamma \supsetneqq \alpha$, the desired conclusion follows.

It remains to prove (2.4). Assertion (i) is obvious, by the definition of $\beta_{p}$ and since $v_{p}$ is composite with $\operatorname{ord}_{\alpha}$. As for (ii), note first that if $\mathfrak{p}$ (resp. $\mathfrak{q}$ ) is the center of $\operatorname{ord}_{\alpha}$ in $\beta_{p}$ (resp. $\gamma$ ), then $\mathfrak{p}$ is a height one prime ideal, whence so is $\mathfrak{q}$ (since $\mathfrak{q} \cap \beta_{p}=\mathfrak{p}$ ). Let $h: R \rightarrow \kappa$ be the canonical map of $R$ onto its residue field $\kappa$. Then $h\left(\beta_{p}\right) \subset h(\gamma) \subset \kappa$. Since $h\left(\beta_{p}\right) \cong \beta_{p} / \mathfrak{p}$ is a discrete valuation ring with fraction field $\kappa$, and $h(\gamma) \cong \gamma / \mathfrak{q} \neq \kappa$, therefore $h(\gamma)=h\left(\beta_{p}\right)$.

Now the valuation rings $V$ which dominate $\gamma$ and are contained in $R$ are in one-one correspondence with the valuation rings in $\kappa$ which dominate $h(\gamma)$, the correspondence being $V \leftrightarrow h(V)[\mathbf{2}$, p. 111, §4.1, Prop. 2]; and since $h(\gamma)$ is a discrete valuation ring, there is a unique such $V$, namely $h^{-1} h(\gamma)$. The corresponding valuation of $K$ dominates $\beta_{p}$ too, and so by uniqueness it must be $v_{p}$. Thus $\gamma \subset V_{p}=V$.

Moreover, $V=h^{-1} h(\gamma)$ has the same residue field as $\gamma$. Also, since $h\left(\mathfrak{m}_{\gamma} V\right)$ is the maximal ideal of $h(\gamma)$ and since the ideals in $V$ are totally ordered, so that $\mathfrak{m}_{\gamma} V$ contains the kernel of $V \rightarrow \gamma$, therefore $\mathfrak{m}_{\gamma} V$ is the maximal ideal of $V$, i.e., $v_{p}\left(\mathfrak{m}_{\gamma}\right)=(0,1)$. 
3. Unique factorization. Before proceeding, we recall a useful device, due to $\mathrm{Du} \mathrm{Val}[\mathbf{3}]$, for representing proximity relations. Fixing a point $\alpha$, consider the proximity matrix

$$
\mathbf{p}=\mathbf{p}(\alpha)=\left(p_{\beta \gamma}\right)_{\beta \supset \alpha, \gamma \supset \alpha}
$$

given by

$$
\begin{aligned}
p_{\beta \gamma} & =1 & & \text { if } \beta=\gamma \\
& =-1 & & \text { if } \beta \prec \gamma \\
& =0 & & \text { otherwise. }
\end{aligned}
$$

Consider also the diagonal matrix $\mathbf{d}=\mathbf{d}(\alpha)=\left(d_{\beta \gamma}\right)_{\beta \supset \alpha, \gamma \supset \alpha}$ given by

$$
\begin{array}{ll}
d_{\beta \gamma} & =0 \quad \text { if } \beta \neq \gamma \\
d_{\beta \beta} & =[\beta: \alpha]
\end{array}
$$

and the refined proximity matrix

$$
\mathbf{d}^{-1} \mathbf{p d}=: \mathbf{P}=\mathbf{P}(\alpha)=\left(P_{\beta \gamma}\right)_{\beta \supset \alpha, \gamma \supset \alpha}
$$

given by

$$
\begin{aligned}
P_{\beta \gamma} & =1 & & \text { if } \beta=\gamma \\
& =-[\gamma: \beta] & & \text { if } \beta \prec \gamma \\
& =0 & & \text { otherwise. }
\end{aligned}
$$

One checks via (1.3) that the matrices $\mathbf{p}$ and $\mathbf{P}$ are column-finite and invertible, and that the entries of $\mathbf{p}^{-1}$ and $\mathbf{P}^{-1}$ are nonnegative integers (cf. also (3.1) and (4.6)).

REMARK. The proximity inequalities for a "column vector" $\mathbf{B}=\left(r_{\beta}\right)$ as in (2.1) can be expressed as $\mathbf{P B} \geq 0$.

Corollary (3.1) (Unique Factorization). For each $\gamma \supset \alpha$ let $\mathbf{e}_{\gamma}$ be the "unit column vector" corresponding to $\gamma$, i.e., the family $\left(e_{\gamma \delta}\right)_{\delta \supset \alpha}$ with $e_{\gamma \delta}=0$ if $\delta \neq \gamma$ and $e_{\gamma \gamma}=1$. Then:

(i) There is a unique $\mathfrak{m}_{\alpha}$-primary complete ideal $\wp_{\gamma}$ with point basis $\mathbf{P}^{-1} \mathbf{e}_{\gamma}$.

(ii) Any $\mathfrak{m}_{\alpha}$-primary complete ideal I can be factored uniquely as

$$
I=\prod_{\gamma \supset \alpha} \wp_{\gamma}^{a_{\gamma}} \quad\left(a_{\gamma} \geq 0\right)
$$

The factorization vector $\mathbf{F}(I):=\left(a_{\gamma}\right)$ is related to the point basis $\mathbf{B}(I)$ of $I$ by

$$
\mathbf{F}(I)=\mathbf{P B}(I) \text {. }
$$

Proof. Since $\mathbf{P P} \mathbf{P}^{-1} \mathbf{e}_{\gamma}=\mathbf{e}_{\gamma} \geq 0$, the existence of $\wp_{\gamma}$ is given by (2.1) (see preceding remark), and its uniqueness by (1.6)(ii).

As mentioned in (1.4), the ideal $\prod \wp_{\gamma}^{a_{\gamma}}$ is complete; and by (1.6)(i), its point basis is $\mathbf{P}^{-1}\left(a_{\gamma}\right)$, which equals $\mathbf{B}(I)$ iff $\left(a_{\gamma}\right)=\mathbf{P B}(I)$, whence the conclusion. 
EXAMPLE (3.2). To each of the ideals $\wp_{\delta}$ associate an adjoint (or conductor-) ideal $\mathfrak{C}_{\delta}$, as follows.

Let $\left(r_{\beta}\right):=\mathbf{P}^{-1} \mathbf{e}_{\delta}$ be the point basis of $\wp_{\delta}$, cf. (3.1). The equation $\mathbf{P}\left(r_{\beta}\right)=\mathbf{e}_{\delta}$ gives, for $\beta \neq \delta$,

$$
r_{\beta}=\sum_{\gamma \succ \beta}[\gamma: \beta] r_{\gamma}
$$

Hence,

$$
\beta \subset \gamma \Longrightarrow r_{\beta} \geq r_{\gamma}
$$

(By induction on the number of points between $\beta$ and $\gamma$ we reduce to where $\gamma$ is a quadratic transform of $\beta$, so that $\gamma \succ \beta$ and we can apply (3.2.1).) Moreover,

$$
r_{\delta}=1, \quad \text { and } \quad r_{\gamma}=0 \text { if } \gamma \nsubseteq \delta .
$$

This is because $\mathbf{P}$ is "upper triangular" $\left(\mathbf{P}_{\beta \gamma}=0\right.$ unless $\left.\beta \subset \gamma\right)$ with 1's on the diagonal, so the same is true of $\mathbf{P}^{-1}$, whose $\delta$-column is $\left(r_{\gamma}\right)_{\gamma \supset \alpha}$.

Now consider the family $\left(r_{\beta}^{\prime}\right)$ given by

$$
\begin{aligned}
r_{\beta}^{\prime} & =r_{\beta}-1 & & \text { if } r_{\beta}>0 \\
& =0 & & \text { if } r_{\beta}=0 ;
\end{aligned}
$$

and set

$$
c_{\beta}:=r_{\beta}^{\prime}-\sum_{\gamma \succ \beta}[\gamma: \beta] r_{\gamma}^{\prime} .
$$

By (3.2.3) and (3.2.2), $r_{\gamma}^{\prime}=0$ unless $\gamma \varsubsetneqq \delta$, and $r_{\gamma}^{\prime}=r_{\gamma}-1$ if $\gamma \subset \delta$. So if $r_{\beta}>1$ then $\beta \neq \delta$ and

$$
\begin{aligned}
& c_{\beta}=\left(r_{\beta}-1\right)-\sum_{\delta \supset \gamma \succ \beta}[\gamma: \beta]\left(r_{\gamma}-1\right) \\
&=\left(r_{\beta}-\sum_{\gamma \succ \beta}[\gamma: \beta] r_{\gamma}\right)-1+\sum_{\delta \supset \gamma \succ \beta}[\gamma: \beta] \\
& \stackrel{(3.2 .1)}{=}-1+\sum_{\delta \supset \gamma \succ \beta}[\gamma: \beta] \geq 0 ;^{3}
\end{aligned}
$$

while if $r_{\beta} \leq 1$ then (3.2.2) implies that $c_{\beta}=0$.

By (2.1) then, there exists a unique complete ideal $\mathfrak{C}_{\delta}$ with point basis $\left(r_{\beta}^{\prime}\right)$, and by (3.1) that ideal is

$$
\mathfrak{C}_{\delta}:=\prod_{\beta \supset \alpha} \wp_{\beta}^{c_{\beta}} .
$$

This $\mathfrak{C}_{\delta}$ has a number of interesting properties, cf. [10], of which we mention only one (conductor property): for every integer $n \geq \operatorname{ord}_{\delta}\left(\mathfrak{C}_{\delta}\right)$, there is a $z \in \alpha$ with $\operatorname{ord}_{\delta}(z)=n$; and if $[\delta: \alpha]=1$ (but not otherwise), then there is no $z \in \alpha$ with $\operatorname{ord}_{\delta}(z)=\operatorname{ord}_{\delta}\left(\mathfrak{C}_{\delta}\right)-1$.

\footnotetext{
${ }^{3}$ Incidentally, (2.4)(ii) yields that $[\gamma: \beta]$ has the same value for all $\gamma$ such that $\delta \supset \gamma \succ \beta$.
} 
4. Simple complete ideals. A simple ideal in $\alpha$ is, by definition, one which cannot be factored non-trivially. A complete $\alpha$-ideal $I$ is simple iff it is not a product of two other complete ideals (for, if $I=J L$ then $I=\bar{J} \bar{L}$ ).

Corollary (4.1). The map $\gamma \mapsto \wp_{\gamma}$ is a one-one correspondence between points infinitely near to $\alpha$ and simple $\mathfrak{m}_{\alpha}$-primary complete ideals. The inverse map takes such a simple ideal to its unique largest base point.

Proof. The first statement follows from (3.1); the second from (3.2.3).

Now we want to vary $\alpha$, so we will write $\wp_{\alpha \gamma}$ instead of $\wp_{\gamma}$. We also set $\wp_{\alpha \delta}=\delta$ whenever $\alpha \nsubseteq \delta$. Note then that for any three points $\alpha \subset \beta$ and $\gamma$,

$$
\operatorname{ord}_{\gamma}\left(\wp_{\alpha \gamma}^{\beta}\right)=\operatorname{ord}_{\gamma}\left(\wp_{\beta \gamma}\right) .
$$

This is clear, from the last assertion in (4.1), if $\beta \nsubseteq \gamma$. Otherwise it just says that the $\beta \gamma$-entries in the inverse refined proximity matrices $\mathbf{P}(\alpha)^{-1}$ and $\mathbf{P}(\beta)^{-1}$ are the same, which holds because $\mathbf{P}(\beta)$ is obtained from $\mathbf{P}(\alpha)$ by chopping off all rows and columns indexed by points not containing $\beta$, so that a similar relation holds between the inverse matrices. (Note that in the calculation of column $\gamma$ of $\mathbf{P}^{-1}$, only those $P_{\beta \gamma}$ for which $\beta \subset \gamma$ come into play, so that in essence we are working with finite upper triangular matrices.) By (1.4), then, $\wp_{\beta \gamma}$ is the integral closure of the transform $\wp_{\alpha \gamma}^{\beta}$. If we use the result that transforms of complete ideals are complete, ([15, p. 381, Prop.5], [7, p. 209, Prop. (6.5)]), then we can conclude that in fact $\wp_{\beta \gamma}=\wp_{\alpha \gamma}^{\beta}$. But just for variety, let us take a brief stroll along another logical path ((4.2) and (4.3)).

Proposition (4.2). For any points $\alpha \subset \beta$ and $\gamma$, the $\beta$-transform of $\wp_{\alpha \gamma}$ is $\wp_{\beta \gamma}$.

Proof. In view of the last assertion in (4.1), we need only consider the case where $\beta \subset \gamma$. Using transitivity of transform (1.5) to induct on the number of points between $\alpha$ and $\beta$, we reduce to where $\beta$ is a quadratic transform of $\alpha$. Then by $\left[\mathbf{9}\right.$, p. 217 , Lemma (2.3)], there exists a simple $\mathfrak{m}_{\alpha}$-primary complete ideal $\wp$ whose point basis outside of $\alpha$ is the same as that of its $\beta$-transform $\wp_{\beta \gamma}$. It follows at once from (4.1) that $\wp=\wp_{\alpha \gamma}$.

Corollary (4.3). For any two points $\alpha \subset \beta$, the $\beta$-transform of any complete finite-colength $\alpha$-ideal is again complete.

PROof. Since transform respects products (1.5), therefore (3.1) reduces us to the case of simple ideals, given by (4.2).

Proposition (4.4). A complete $\mathfrak{m}_{\alpha}$-primary ideal $I$ is divisible by $\wp_{\gamma}$ iff $\operatorname{ord}_{\gamma}$ is a Rees valuation of $I$.

Proof. Using the fact that a local ring dominates the blowup of a product of ideals iff it dominates the blowup of each of the factors, we reduce readily 
to where $I=\wp_{\gamma}$, in which case the assertion is that $(*)$ : $\operatorname{ord}_{\gamma}$ is the unique Rees valuation of $\wp_{\gamma}$, which is shown in [7, p. 245, Prop. (21.3)], or, in a more elementary way, in $[6, \text { p. 333, Thm. 4.2]. })^{4}$

We define the valuation vector $\mathbf{V}(I)$ of an $\alpha$-ideal $I$ to be the column vector $\left(\operatorname{ord}_{\beta}(I)\right)_{\beta \supset \alpha}$. Though $\mathbf{V}(I)$ has infinitely many nonzero entries, it can still be premultiplied by a row-finite matrix, for example by the transpose $\mathbf{p}^{\mathbf{t}}$ of $\mathbf{p}$.

Proposition (4.5). For any $\alpha$-ideal I,

$$
\mathbf{B}(I)=\mathbf{p}^{\mathbf{t}} \mathbf{V}(I) .
$$

Proof. The proposition states that for any $\beta \supset \alpha$,

$$
\operatorname{ord}_{\beta}\left(I^{\beta}\right)=\operatorname{ord}_{\beta}(I)-\sum_{\alpha \subset \gamma \prec \beta} \operatorname{ord}_{\gamma}(I),
$$

which is an immediate consequence of the relation $I \beta=t^{c} u^{d} I^{\beta}$ in (1.5).

Corollary (4.6). The entries of the matrix $\mathbf{p}^{-1}$ are

$$
\begin{aligned}
\left(\mathbf{p}^{-1}\right)_{\beta \gamma} & =\operatorname{ord}_{\gamma}\left(\mathfrak{m}_{\beta}\right) & & \text { if } \beta \subset \gamma \\
& =0 & & \text { otherwise. }
\end{aligned}
$$

Proof. If $\beta \subset \gamma$, then after chopping off some rows and columns from $\mathbf{p}$ we may, as in the remarks preceding (4.2), assume that $\beta=\alpha$, and then just take $I=\mathfrak{m}_{\alpha}$ in (4.5) (or at least in the equivalent relation $\mathbf{V}(I)=\left(\mathbf{p}^{-1}\right)^{\mathbf{t}} \mathbf{B}(I)$ ). The second equality results from the corresponding property of $\mathbf{p}$.

Remarks. (1) The formulation $\mathbf{V}(I)=\left(\mathbf{p}^{-1}\right)^{\mathbf{t}} \mathbf{B}(I)$ of (4.5), i.e., by (4.6),

$$
\operatorname{ord}_{\beta}(I)=\sum_{\alpha \subset \gamma \subset \beta} \operatorname{ord}_{\beta}\left(\mathfrak{m}_{\gamma}\right) \operatorname{ord}_{\gamma}\left(I^{\gamma}\right)
$$

is a special case of $[\mathbf{9}$, p. 209, Lemma (1.11)], cf. proof of (2.3) above.

\footnotetext{
${ }^{4}$ I learned only recently (April, 1993) that $(*)$ is essentially contained in Hironaka's 1960 Harvard thesis (Chapter 1, §4, Thm. 10). When I mentioned (*) to Zariski in the early 1970's, he seemed unfamiliar with it, but quickly came up with the following proof. Proceed by induction on the number of base points of $\gamma$, the assertion being obvious when that number is 1 (i.e., $\gamma=\alpha$ and $\wp_{\gamma}=\mathfrak{m}_{\alpha}$ ). So assume $\wp:=\wp_{\gamma} \neq \mathfrak{m}_{\alpha}$, and let $Y$ be the scheme over $\operatorname{Spec}(\alpha)$ obtained by first blowing up $\mathfrak{m}_{\alpha}$ and then blowing up the transform $\wp_{\beta \gamma}$ of $\wp_{\gamma}$ in the unique quadratic transform $\beta$ of $\alpha$ dominated by $\gamma$, cf. (4.2). It results from the inductive hypothesis that the only valuations which dominate $\alpha$ and whose centers on $Y$ are one-dimensional are $\operatorname{ord}_{\alpha}$ and $\operatorname{ord}_{\gamma}$. It suffices therefore to show that the center of $\operatorname{ord}_{\alpha}$ on the blowup $X$ of $\wp$ is 0 -dimensional. But the equality $s(\wp)=\operatorname{ord}_{\alpha}(\wp)$ preceding (2.3) implies that $\wp$ has a basis $\left(x_{0}, x_{1}, \ldots, x_{n}\right)$ with $\operatorname{ord}_{\alpha}\left(x_{i}\right)>\operatorname{ord}_{\alpha}\left(x_{0}\right)$ for all $i>0$, i.e., $x_{i} / x_{0}$ lies in the maximal ideal of the valuation ring of $\operatorname{ord}_{\alpha}$, so that the local ring on $X$ dominated by $\operatorname{ord}_{\alpha}$ has the same residue field as $\alpha$.
} 
(2) The fact that the identity matrix $\mathbf{p p}^{-1}$ has zero entries off the diagonal translates to the relation

$$
\operatorname{ord}_{\gamma}\left(\mathfrak{m}_{\beta}\right)=\sum_{\beta \prec \delta \subset \gamma} \operatorname{ord}_{\gamma}\left(\mathfrak{m}_{\delta}\right) \quad(\beta \varsubsetneqq \gamma) .
$$

(3) Replacing $\mathbf{p}$ by $\mathbf{P}$, we find similarly that

$$
\operatorname{ord}_{\beta}\left(\wp_{\beta \gamma}\right)=\sum_{\beta \prec \delta \subset \gamma}[\gamma: \beta] \operatorname{ord}_{\delta}\left(\wp_{\delta \gamma}\right) \quad(\beta \varsubsetneqq \gamma) .
$$

(These last two equations transform into each other by reciprocity, cf. (4.8).)

Next we define the intersection number $(I \cdot J)=(J \cdot I)$ of two finite-colength $\alpha$-ideals to be

$$
(I \cdot J):=\sum_{\beta \supset \alpha}[\beta: \alpha] \operatorname{ord}_{\beta}\left(I^{\beta}\right) \operatorname{ord}_{\beta}\left(J^{\beta}\right)=\mathbf{B}(I)^{\mathbf{t}} \mathbf{d B}(J) .
$$

Corollary (4.7). For any $\alpha$-ideal I and any $\beta \supset \alpha$,

$$
\left(I \cdot \wp_{\beta}\right)=[\beta: \alpha] \operatorname{ord}_{\beta}(I) .
$$

Proof. $\mathbf{B}\left(\wp_{\beta}\right)$ is the $\beta$-column of $\mathbf{P}^{-1}=\mathbf{d}^{-1} \mathbf{p}^{-1} \mathbf{d}$, so we have equal row vectors

$$
\left(\left(I \cdot \wp_{\beta}\right)\right)_{\beta \supset \alpha}=\mathbf{B}(I)^{\mathbf{t}} \mathbf{d} \mathbf{P}^{-1}=\mathbf{B}(I)^{\mathbf{t}} \mathbf{p}^{-1} \mathbf{d}^{(4.5)} \stackrel{V}{=}(I)^{\mathbf{t}} \mathbf{p} \mathbf{p}^{-1} \mathbf{d}=(\mathbf{d} \mathbf{V}(I))^{\mathbf{t}},
$$

whence the conclusion.

Corollary (4.8) (Reciprocity). ${ }^{5}$ For any $\beta \supset \alpha, \gamma \supset \alpha$,

$$
[\beta: \alpha] \operatorname{ord}_{\beta}\left(\wp_{\gamma}\right)=[\gamma: \alpha] \operatorname{ord}_{\gamma}\left(\wp_{\beta}\right) .
$$

Proof. $\left(\wp_{\gamma} \cdot \wp_{\beta}\right)=\left(\wp_{\beta} \cdot \wp_{\gamma}\right)$.

Given two vectors $\mathbf{B}=\left(r_{\beta}\right)_{\beta \supset \alpha}, \mathbf{B}^{\prime}=\left(r_{\beta}^{\prime}\right)_{\beta \supset \alpha}$, we write $\mathbf{B} \geq \mathbf{B}^{\prime}$ to signify that $r_{\beta} \geq r_{\beta}^{\prime}$ for all $\beta$.

Corollary (4.9). For any two finite-colength complete $\alpha$-ideals $I$ and $J$,

$$
\mathbf{B}(I) \geq \mathbf{B}(J) \Longrightarrow J \supset I .
$$

Proof. According to (4.6), the entries of $\left(\mathbf{p}^{\mathbf{t}}\right)^{-1}$ are all $\geq 0$, so by (4.5), $\mathbf{B}(I) \geq \mathbf{B}(J) \Longrightarrow \mathbf{V}(I) \geq \mathbf{V}(J)$, whence the conclusion.

\footnotetext{
${ }^{5}$ [7, p. 247, Prop. (21.4)], [6, p. 334, Thm. 4.3].
} 
Corollary $(4.10) .{ }^{6}$ If $\delta \subset \gamma$ then $\wp_{\delta} \supset \wp_{\gamma}$.

Proof. By an obvious induction, we may assume that $\gamma$ is a quadratic transform of $\delta$, so that $\delta \prec \gamma$. Then the matrix equation $\mathbf{P}^{-1} \mathbf{P}=\mathbf{1}$ yields, for $\alpha \subset \beta \varsubsetneqq \gamma$,

$$
\operatorname{ord}_{\beta}\left(\wp_{\gamma}^{\beta}\right)=\sum_{\alpha \subset \delta^{\prime} \prec \gamma}\left[\gamma: \delta^{\prime}\right] \operatorname{ord}_{\beta}\left(\wp_{\delta^{\prime}}^{\beta}\right) \geq \operatorname{ord}_{\beta}\left(\wp_{\delta}^{\beta}\right),
$$

whence by (3.2.3), $\mathbf{B}\left(\wp_{\gamma}\right) \geq \mathbf{B}\left(\wp_{\delta}\right)$, and we can apply (4.9).

The next result gives a characterization of proximity for points containing $\alpha$ in terms of their corresponding simple complete ideals - a characterization found by D. B. Scott and used by Hoskin in [5, $\S 3]$ —namely $\beta \succ \gamma \Leftrightarrow \wp_{\gamma}$ divides $\mathfrak{q}_{\beta}$, the smallest $\operatorname{ord}_{\beta}$-ideal strictly containing $\wp_{\beta}$. Note however that our result says more; and besides being valid in arbitrary two-dimensional regular local rings, it also shows that $\mathfrak{q}_{\beta}$ is actually the smallest complete ideal strictly containing $\wp_{\beta}$. (This has been proved previously by Noh [12, Thm. 3.1], at least when the residue field of $\alpha$ is algebraically closed.)

TheOREm (4.11). Let $\beta \supset \alpha$. Among complete ideals I in $\alpha$ strictly containing $\wp_{\beta}$, there is a smallest, viz.

$$
\mathfrak{q}_{\beta}:=\prod_{\alpha \subset \gamma \prec \beta} \wp_{\gamma}^{[\beta: \gamma]} .
$$

For any valuation $v$ dominating $\beta, \mathfrak{q}_{\beta}$ is even a v-ideal $\left(v(x) \geq v\left(\mathfrak{q}_{\beta}\right) \Rightarrow x \in \mathfrak{q}_{\beta}\right)$.

Proof. To prove that $\mathfrak{q}_{\beta} \subset I$, it's enough to show that $w\left(\mathfrak{q}_{\beta}\right) \geq w(I)$ for every Rees valuation $w=\operatorname{ord}_{\delta}$ of $I$, cf. (1.4); so it's certainly enough that $w\left(\mathfrak{q}_{\beta}\right) \geq w\left(\wp_{\beta}\right)$. Now every $\wp_{\gamma}$ dividing $I$ strictly contains $\wp_{\beta}$, and so by (4.10), $\beta \nsubseteq \gamma$, and by (4.1), $\beta$ is not a base point of $\wp_{\gamma}$, i.e., $\left(\wp_{\gamma}\right) \beta$ is a principal ideal. Thus $I \beta$ is a principal ideal, so $\beta$ dominates a local ring $S$ on the blowup of $I$, and consequently $\delta \nsupseteq \beta$ (otherwise $S$, being dominated by the valuation ring of ord ${ }_{\delta}$ - which is also a local ring on the blowup - would have to be that valuation ring, and so could not be contained in $\beta$ ). The first assertion follows then from:

Lemma (4.12). For any $\delta \supset \alpha$, we have

$$
\begin{aligned}
\operatorname{ord}_{\delta}\left(\mathfrak{q}_{\beta}\right) & =\operatorname{ord}_{\delta}\left(\mathfrak{p}_{\beta}\right) & & \text { if } \delta \nsupseteq \beta \\
& =\operatorname{ord}_{\delta}\left(\mathfrak{p}_{\beta}\right)-\operatorname{ord}_{\delta}\left(\mathfrak{m}_{\beta}\right) & & \text { if } \delta \supset \beta .
\end{aligned}
$$

In particular, $\operatorname{ord}_{\delta}\left(\mathfrak{q}_{\beta}\right) \leq \operatorname{ord}_{\delta}\left(\mathfrak{p}_{\beta}\right)$ for all $\delta$, and so $\mathfrak{q}_{\beta} \supsetneqq \wp_{\beta}$.

Proof. By definition, $\mathbf{F}\left(\wp_{\beta}\right)-\mathbf{F}\left(\mathfrak{q}_{\beta}\right)$ is column $\beta$ of the matrix $\mathbf{P}$. Hence by $(3.1), \mathbf{B}\left(\wp_{\beta}\right)-\mathbf{B}\left(\mathfrak{q}_{\beta}\right)$ is column $\beta$ of $\mathbf{P}^{-1} \mathbf{P}=\mathbf{1}$, and so by $(4.5), \mathbf{V}\left(\wp_{\beta}\right)-\mathbf{V}\left(\mathfrak{q}_{\beta}\right)$ is column $\beta$ of $\left(\mathbf{p}^{\mathbf{t}}\right)^{-1}$. The conclusion results then from (4.6).

\footnotetext{
${ }^{6}$ Cf. $[\mathbf{1 5}$, p. $392,($ F) $]$.
} 
For the second assertion in (4.11), set

$$
I:=\left\{x \in \alpha \mid v(x) \geq v\left(\mathfrak{q}_{\beta}\right)\right\} \supset \mathfrak{q}_{\beta} \supsetneqq \wp_{\beta},
$$

so that as above $I \beta$ is principal, say $I \beta=z \beta$. The kernel $J$ of the homomorphism taking $x \in I$ to $\left(x z^{-1}+\mathfrak{m}_{\beta}\right) \in \beta / \mathfrak{m}_{\beta}$ consists of all $x \in I$ such that $v\left(x z^{-1}\right)>0$, i.e., $v(x)>v\left(\mathfrak{q}_{\beta}\right)$; so $J$ is a complete ideal not containing $\mathfrak{q}_{\beta}$. But $J$ does contain $\mathfrak{p}_{\beta}$, because $z^{-1} \mathfrak{p}_{\beta} \beta$ is a non-principal $\beta$-ideal, so that $z^{-1} \mathfrak{p}_{\beta} \beta \subset \mathfrak{m}_{\beta}$; and so by the first part of (4.11), $J=\wp_{\beta}$. Thus we have an injective $\alpha / \mathfrak{m}_{\alpha^{-}}$ linear map $I / \mathfrak{p}_{\beta} \hookrightarrow \beta / \mathfrak{m}_{\beta}$, whence

$$
\lambda_{\alpha}\left(I / \wp_{\beta}\right) \leq[\beta: \alpha] .
$$

(Recall that $\lambda$ denotes length.) But the point bases of $\wp_{\beta}$ and $\mathfrak{q}_{\beta}$ are identical except for a 1 at $\beta$, cf. proof of (4.12). So by the Hoskin-Deligne length formula [9, p. 222, Thm. (3.1)],

$$
\lambda_{\alpha}\left(\mathfrak{q}_{\beta} / \wp_{\beta}\right)=[\beta: \alpha] .
$$

Since $\mathfrak{q}_{\beta} \subset I$, we conclude that $\mathfrak{q}_{\beta}=I$.

5. Valuations and proximity. To a valuation $v$ dominating $\alpha$, associate the sequence

$$
\alpha=\alpha_{0} \subset \alpha_{1} \subset \cdots \subset \alpha_{i} \subset \alpha_{i+1} \subset \cdots
$$

where for each $i \geq 0, \alpha_{i+1}$ is the unique quadratic transform of $\alpha_{i}$ dominated by $v$. As in (1.2), the sequence $(5.1)_{v}$ terminates after finitely many steps iff $v$ is residually transcendental over $\alpha$. Assume from here on that the sequence is infinite, i.e., $v$ is residually algebraic (or "zero-dimensional") over $\alpha$. Note, conversely, that any infinite sequence $\left(\beta_{i}\right)_{i \geq 0}$ of successive quadratic transforms is associated to a unique zero-dimensional valuation, namely the one having valuation ring $\cup_{i} \beta_{i}$. Thus a zero-dimensional valuation dominating $\alpha$ can be identified with a maximal branch running through the tree of points infinitely near to $\alpha$.

In some sense, $v$ is the limit of the valuations $\operatorname{ord}_{\alpha_{i}}$. And, as will now be briefly discussed, the proximity relations among the $\alpha_{i}$-as encoded, say, in the matrix $\mathbf{P}_{v}$ obtained from the refined proximity matrix $\mathbf{P}(\alpha)$ by restricting to those entries $P_{\beta \gamma}$ for which both $\beta$ and $\gamma$ are in (5.1) $)_{v}$-determine many of the basic properties of $v$.

For instance, $\mathbf{P}_{v}$ determines the rank and rational rank of $v,{ }^{7}$ and in case of rational rank 1 , whether $v$ is discrete or not. In essence, this is shown in $[\mathbf{1 4}, \S 9]{ }^{8}$

\footnotetext{
${ }^{7}$ except when $\alpha$ is not complete, $v$ is residually finite over $\alpha$, and there are only finitely many "satellite" points in $(5.1)_{v}$, i.e., points proximate to two others in $(5.1)_{v}$ : the problem is that a rank-two discrete valuation of the completion of $\alpha$ can restrict to a rank-one discrete valuation of $\alpha$ with the same proximity matrix, cf. [14, p. 118, Example 3.5].

${ }^{8}$ Spivakovsky represents proximity relations via dual graphs of closed fibers on smooth birational $\operatorname{Spec}(\alpha)$-schemes. (See also [3] for this technique.) An equivalent, but more direct and very effective graphical representation was invented by Enriques: it is the "Enriques diagram," a structured version of (5.1) $v$ obtained by grouping maximal sets of points proximate
} 
(5.2). So let us indicate how, in analogy with the classical theory of plane curve singularities, we can use $\mathbf{P}_{v}$ to analyze $v$ more closely.

ExAmple. Suppose that $\alpha$ is complete (or at least henselian). Let $f_{0}$ be an irreducible element in $\alpha_{0}:=\alpha$; and having inductively defined $f_{i} \in \alpha_{i}$, let $\alpha_{i+1}$ be the unique quadratic transform of $\alpha_{i}$ in which the proper transform $f_{i+1}$ of $f_{i}$ is a non-unit. The $\alpha_{i}$ are "the points infinitely near to $\alpha$ lying on $f$."

This sequence of points determines a rank-two composed valuation $v=v_{1} \circ v_{2}$, where the valuation ring of $v_{1}$ is the integral closure of $\alpha / f_{0}$. The singularity invariants of $f_{0}$ (multiplicity sequence, value semigroup, ... ) are all calculable from $\mathbf{P}_{v}$. Here $\mathbf{P}_{v}$ carries only a finite amount of information, because for large $i$, $f_{i}$ is of order one in $\alpha_{i},\left[\alpha_{i+1}: \alpha_{i}\right]=1$, and there is just one $\alpha_{j}$ (namely $\alpha_{i+1}$ ) proximate to $\alpha_{i}$.

Now consider an arbitrary zero-dimensional valuation $v$, let $(5.1)_{v}$ be the corresponding quadratic sequence, and set

$$
\mathfrak{m}_{i}:=\mathfrak{m}_{\alpha_{i}}, \quad e_{i}:=\left[\alpha_{i}: \alpha\right] .
$$

Consider also the sequence of $v$-ideals defined inductively by

$$
J_{0}=\alpha, \quad J_{i+1}=\left\{x \in \alpha \mid v(x)>v\left(J_{i}\right)\right\} .
$$

These $J_{i}$ are all the finite-colength $v$-ideals. We have then four sequences:

(1) The multiplicity sequence $\left(v\left(\mathfrak{m}_{0}\right) / v\left(\mathfrak{m}_{i}\right), e_{i}\right)_{1 \leq i<\infty}$.

[The quotients $\left(v\left(\mathfrak{m}_{0}\right) / v\left(\mathfrak{m}_{i}\right)\right.$ are rational numbers in the interval $[1, \infty)$.]

(2) The semigroup-length sequence $\left(v\left(J_{i}\right) / v\left(\mathfrak{m}_{0}\right), \lambda_{\alpha}\left(\alpha / J_{i}\right)\right)_{1 \leq i<\infty}$.

[The quotients $\left(v\left(J_{i}\right) / v\left(\mathfrak{m}_{0}\right)\right.$ are rational numbers in the interval $[1, \infty)$.]

(3) The point basis sequence $\mathbf{B}\left(J_{i}\right)_{1 \leq i<\infty}$.

[The base points of any $J_{i}$ are among the $\alpha_{j}$, so that $\mathbf{B}\left(J_{i}\right)$ may be represented in the form $\left(b_{i j}\right)_{j \geq 0}$.]

(4) The factorization sequence $\mathbf{F}\left(J_{i}\right)_{1 \leq i<\infty}$.

[The simple complete ideals dividing any $J_{i}$ are among the $\wp_{j}$ corresponding to the points $\alpha_{j}$ in (5.1) $v_{v}$-and for each $j \geq 0, \wp_{j}$ is a $v$-ideal-so that $\mathbf{F}\left(J_{i}\right)$ may be represented in the form $\left(a_{i j}\right)_{j \geq 0}$.]

TheOREM. The proximity matrix $\mathbf{P}_{v}$ determines each one of the preceding four sequences, and vice-versa.

The proof begins with the observation that $v$ can be replaced by its "approximations" $\operatorname{ord}\left(\alpha_{i}\right)(i \rightarrow \infty)$. The analysis for ord $\left(\alpha_{i}\right)$ is in many respects closely related to that of the singularity at the origin of the "plane curve" $f_{i}=0$, where $f_{i}$ is a sufficiently general element of the corresponding simple complete ideal $\wp_{i}$. (For some results along these lines, cf. [11] and [14].)

Details may appear elsewhere.

to the same one (when those sets contain more than one member) successively along alternating horizontal and vertical lines, cf. [4, Chap. 1, §8, pp. 374-381]. 
Remark (added in proof). Here is another more geometric way of looking at the proximity inequalities of Theorem (2.1).

Let $I$ be a finite-colength $\alpha$-ideal, and let $f: X \rightarrow \operatorname{Spec}(\alpha)$ be a proper birational map such that $X$ is non-singular and $\mathcal{I}:=I \mathcal{O}_{X}$ is invertible. Then each irreducible component $E$ of the closed fibre $f^{-1}\left(\mathfrak{m}_{\alpha}\right)$ is a projective line over the field $\alpha / \mathfrak{m}_{\alpha}$, and the restriction $\left.\mathcal{I}\right|_{E}$ is an invertible $\mathcal{O}_{E}$-module generated by global sections; so the intersection number $(\mathcal{I} \cdot E)$ (i.e., the degree of $\left.\left.\mathcal{I}\right|_{E}\right)$ is $\geq 0$. But this intersection number is precisely $[\beta: \alpha]\left(r_{\beta}-\sum_{\gamma \succ \beta}[\gamma: \beta] r_{\gamma}\right)$ where $\beta \supset \alpha$ is the unique point such that the valuation ring of $\operatorname{ord}_{\beta}$ is the local ring of the generic point of $E$ on $X$. The proof, suitably generalized, leads further to higher-dimensional proximity inequalities, cf. [16, p. 988, Cor. 4].

The converse ("if") part of Theorem (2.1) also results from the preceding interpretation, basically because any invertible $\mathcal{O}_{X}$-module $\mathcal{J}$ with $(\mathcal{J} \cdot E) \geq 0$ for all $E$ must be of the form $J \mathcal{O}_{X}$ for some $\alpha$-ideal $J$ [7, p. 210, Thm. (12.1)].

\section{REFERENCES}

1. S. S. Abhyankar, On the valuations centered in a local domain, Amer. J. Math 78 (1956), 321-348.

2. N. Bourbaki, Algèbre Commutative, chap. 5-6, Act. Sci. et Ind., no. 1308, Hermann, Paris, 1964.

3. P. Du Val, On isolated singularities of surfaces which do not affect the conditions of adjunction, Proc. Cambridge Phil. Soc. 30 (1934), 453-459.

4. F. Enriques and O. Chisini, Lezioni sulla teoria geometrica delle equazioni e delle funzioni algebriche, vol. II, N. Zanichelli, Bologna, 1918.

5. M. A. Hoskin, Zero-dimensional valuation ideals associated with plane curve branches, Proc. London Phil. Soc. (3) 6 (1956), 70-99.

6. C. Huneke, Complete ideals in two-dimensional regular local rings, Commutative Algebra, Proceedings of a Microprogram held June 15-July 2, 1987, Springer-Verlag, New York, 1989, pp. 325-337.

7. J. Lipman, Rational singularities, with applications to algebraic surfaces and unique factorization, Publ. Math. Inst. Hautes Études Sci. 36 (1969), 195-279.

8. _ Appendix to Chapter II, Algebraic Surfaces (2nd edition), by O. Zariski, SpringerVerlag, New York, 1971, pp. 45-50.

9. - On complete ideals in regular local rings, Algebraic Geometry and Commutative Algebra, vol. I, in honor of Masayoshi Nagata, Kinokuniya, Tokyo, 1988, pp. 203-231.

10. - Adjoints and polars of simple complete ideals in two-dimensional regular local rings, Algebra and Algebraic Geometry, Tenerife, June 1992, Proceedings (to appear).

11. S. Noh, Sequence of valuation ideals of prime divisors of the second kind in 2-dimensional regular local rings, J. Algebra (to appear).

12. S. Noh, Adjacent integrally closed ideals in dimension two, J. Pure and Applied Algebra (to appear).

13. D. G. Northcott, Abstract dilatations and infinitely near points, Proc. Cambridge Phil. Soc. 52, Part 2 (1956), 176-197.

14. M. Spivakovsky, Valuations in function fields of surfaces, Amer. J. Math 112 (1990), 107-156.

15. O. Zariski and P. Samuel, Commutative Algebra, vol. 2, D. van Nostrand, Princeton, 1960.

16. A. Campillo, G. Gonzalez-Sprinberg, and M. Lejeune-Jalbert, Amas, idéaux à support fini et chaines toriques, C. R. Acad. Sci. Paris, Série I, 315 (1992), 987-990.

Department of Mathematics, Purdue University, W. Lafayette, IN 47907, USA

E-mail address: lipman@math.purdue.edu 